\title{
EXAMPLES OF SELF-DUAL, EINSTEIN METRICS OF $(2,2)$-SIGNATURE
}

\author{
NOVICA BLAŽIĆ* and SRDJAN VUKMIROVIĆ*
}

\begin{abstract}
In this paper we use para-quaternionic reduction to construct a family of examples of self-dual Einstain metrics of neutral signature, which are not Ricci flat, nor locally homogenous. The curvature of these manifolds is studied in detail and these examples are compared with the orbifolds $\mathcal{O}_{p, q}(1)$ given by Galicki and Lawson. Particular attention is given to the sign and to the pinching of the sectional curvatures.
\end{abstract}

\section{Introduction}

In 4 dimensions, self-dual manifolds are important not only in Riemannian geometry, i.e. signature $(0,4)$, but also in Kleinian (or neutral) geometry, i.e. in signature $(2,2)$. We refer to [8] where examples of Einstein, self-dual neutral signature metrics are given. However, non-trivial examples of non-Ricci flat, Einstein self-dual manifolds were not known previously. We refer to [6] and [7] where families of such examples were constructed in the Riemannian setting. Let $p$ and $q$ be coprime distinct positive integers. In the present note, motivated by the examples cited above, we shall construct a family of neutral signature manifolds $M_{p, q}$ which are not locally homogenous.

The quaternions are the Clifford algebra $C(2)$ and the para-quaternions are the Clifford algebra $C(1,1)$. As remarked in [9], quaternionic reduction can be applied not only in the Riemannian but also in the pseudo-Riemannian cathegory. We adopt this approach and use para-quaternions rather than quaternions for two reasons. First, because the curvature tensor of self-dual manifolds has many properties in common both with the quaternionic and also the para-quaternionic manifolds - see [1], [6], [7] for further details. Second, because the Clifford algebra $C(1,1)$ provides a natural framework for describing metrics of neutral signatures.

It is interesting to compare this construction with the example, given in [7], which was obtained by quaternionic reduction. In both cases, an algebraic submanifold $\mathscr{K}_{0}$ of the projective planes $\left(\mathrm{H} P^{2}\right.$ or $\left.\tilde{\mathrm{H}} P^{2}\right)$ was constructed.

\footnotetext{
* Research partially supported by SFS, project \#0401.
}

Received August 8, 2002. 
In the quaternionic (positive definite) case, $\mathscr{K}=\mathscr{K}_{0}$ was a differentiable, complete, submanifold. In the para-quaternionic (indefinite) case, $\mathscr{K}_{0}$ was not a differentiable submanifold. The singular set $\mathscr{S}$ lay on a real hyperquadric in $\tilde{\mathrm{H}} P^{2}$. Let $\mathscr{K}=\mathscr{K}_{0} \backslash \mathscr{S}$. In both cases, the family of isometric actions $\phi_{p, q}, \quad p, q \in \mathrm{N}, \quad(p, q)=1$ of a group $G\left(S^{1}\right.$ or R $)$ on five-dimensional submanifold $\mathscr{K}$ was defined. In the quaternionic case, the action was locally free action; in the para-quaternionic case, the actions $\phi_{p, q}$ were free. In the quaternionic case $\mathscr{K} / G$ was a Riemannian orbifold, while in the para-quaternionic case, $\mathscr{K} / G$ was an incomplete neutral signature manifold.

Here is a brief outline to this paper. In $\S 2$, we deal with the para-quaternions. We shall discuss the elementary properties of the Clifford algebra $\tilde{\mathrm{H}}=C(1,1)$ $=C(0,2)$ and of the associated projective plane $\tilde{\mathrm{H}} P^{2}$. In $\S 3$ we shall define the action of the group $G=\mathrm{R}$ on $\tilde{\mathrm{H}} P^{2}$ and on the algebraic submanifold $\mathscr{K}_{0}$ of $\tilde{\mathrm{H}} P^{2}$. We shall also study the basic geometry of the orbit space $\mathscr{K}_{0} / G$ which are equiped with the metric $g_{p, q}$ induced by the submersion $\mathscr{K}_{0} \rightarrow \mathscr{K}_{0} / G$.

In $\S 4$ we shall compute the sectional curvature and the Jacobi curvature operator of the metric $g_{p, q}$ in terms of the para-quaternions. We use this description to prove in $\S 5$ that $g_{p, q}$ is a self-dual, Einstein metric which is pointwise Osserman. We shall also show that this metric is not even locally homogeneous. Let $\mathscr{O}_{p, q}(1)$ be the orbifolds $\mathscr{O}_{p, q}(1)$ defined previously by Galicki and Lawson [6], [7]. We conclude in $\S 6$ by applying the methods we have derived in previous sections to study the curvature tensor of these manifolds in more detail.

We are gratefull to Peter Gilkey and the referee for various suggestions and comments.

\section{Preliminaries}

The paraquaternions are the real unital Clifford algebra $\tilde{\mathrm{H}}:=C(1,1)=$ $C(0,2)$ which is generated by elements $i, j, k$ subject to the relations:

$$
i^{2}=-\epsilon_{1}=-1, \quad j^{2}=-\epsilon_{2}=1, \quad k^{2}=-\epsilon_{3}=1, \quad i j=k=-j i .
$$

Let $q:=x+y i+z j+w k$. The conjugate $\bar{q}$, the real part $\Re q$, the imaginary part $\Im q$, and the square norm $|\cdot|^{2}$ are then defined by:

$$
\begin{aligned}
\bar{q} & :=x-y i-z j-w k, \\
\Re q & :=x, \quad \Im q:=y i+z j+w k, \\
|q|^{2} & :=q \bar{q}=x^{2}+y^{2}-z^{2}-w^{2} .
\end{aligned}
$$

The norm is multiplicative, i.e. it satisfies the relationship

$$
\left|q_{1} q_{2}\right|^{2}=\left|q_{1}\right|^{2}\left|q_{2}\right|^{2} .
$$


Notice that the pseudosphere of signature $(2,1)$ of all unit spacelike paraquaternions

$$
S^{2,1}=\tilde{\mathrm{H}}_{1}:=\left\{\left.q \in \mathrm{H}|| q\right|^{2}=1\right\}
$$

is isomorphic to the Lie group $S U(1,1)$. Give $\tilde{\mathrm{H}}^{3}$ the pseudo-Riemannian metric of signature $(6,6)$ defined by

$$
g(u, v):=\Re\left(\bar{u}_{1} v_{1}+\bar{u}_{2} v_{2}+\bar{u}_{3} v_{3}\right)=\Re(\bar{u} \cdot v)
$$

for all $u=\left(u_{1}, u_{2}, u_{3}\right), \quad v=\left(v_{1}, v_{2}, v_{3}\right)$ in $\tilde{\mathrm{H}}^{3}$. Let $S^{6,5}$ be the associated pseudosphere of signature $(6,5)$. The para-quaternionic projective plane $\tilde{\mathrm{H}} P^{2}$ is defined as the set of equivalence classes

$$
\begin{aligned}
\tilde{\mathrm{H}} P^{2}:=\left\{[u] \mid u \in S^{6,5}\right\} / \sim \\
\quad \text { where } \quad u=\left(u_{0}, u_{1}, u_{2}\right) \sim\left(u_{0} h, u_{1} h, u_{2} h\right)=u h, h \in \tilde{\mathrm{H}}_{1} .
\end{aligned}
$$

Let $\pi(u):=[u]$ be the natural projection from $S^{6,5}$ to $\tilde{\mathrm{H}} P^{2}$. The associated vertical subspace $\mathscr{V}_{u} \subset T_{u} S^{6,5} \subset T_{u} \tilde{\mathrm{H}}^{3} \cong \tilde{\mathrm{H}}^{3}$ of the submersion $\pi$ at the point $u \in S^{6,5}$ is generated by the vectors $u i, u j, u k$ i.e.

$$
\mathscr{V}_{u}=\mathrm{R}\langle u i, u j, u k\rangle .
$$

Moreover, the orthogonal decomposition with respect to the metric $g$

$$
T_{u} S^{6,5}=\mathscr{V}_{u} \oplus \mathscr{H}_{u}
$$

into vertical and horizontal subspace is $\tilde{\mathrm{H}}_{1}$ invariant. We usually fix a local horizontal section $u \subset S^{6,5}$ over $\tilde{\mathrm{H}} P^{2}$. This allows us to indentify

$$
T_{[u]} \tilde{\mathrm{H}} P^{2} \cong \mathscr{H}_{u} \quad \text { by } \quad T_{[u]} \tilde{\mathrm{H}} P^{2} \ni X \leftrightarrow \tilde{X} \in \mathscr{H}_{u} \subset \tilde{\mathrm{H}}^{3},
$$

where $\tilde{X}$ is the unique horizontal lift of the vector $X$. Right multiplication of vectors from $\tilde{\mathrm{H}}^{3}$ by $i, j, k$ preserves the horizontal subspace $\mathscr{H}_{u}$. Consequently, we can locally define the endomorphisms

$$
J_{1}(X):=\pi_{*}(\tilde{X} i), \quad J_{2}(X):=\pi_{*}(\tilde{X} j), \quad J_{3}(X):=\pi_{*}(\tilde{X} k)
$$

where the vectors $X$ and $\tilde{X}$ are related as above. They satisfy the same relations as $i, j$ and $k$, i.e. if $(\alpha, \beta, \gamma)$ is any cyclic permutation of $(1,2,3)$, then we have

$$
J_{\alpha}^{2}=-\epsilon_{\alpha}, \quad J_{\alpha} J_{\beta}=-\epsilon_{\gamma} J_{\gamma} .
$$

Although the definition of $J_{1}, J_{2}$ and $J_{3}$ depends on the section $u$ over $\tilde{\mathrm{H}} P^{2}$, the subbundle $\mathrm{R}\left\langle J_{1}, J_{2}, J_{3}\right\rangle$ of $\operatorname{End}\left(T \tilde{\mathrm{H}} P^{2}\right)$ is globally defined. This is 
a pseudo-Riemannian analogue of the notion of a quaternionic Kähler structure on quaternionic projective plane $\mathrm{H} P^{2}$. The submersion induces a pseudoRiemannian metric

$$
\langle X, Y\rangle=g(\tilde{X}, \tilde{Y})
$$

of signature $(4,4)$ on $\tilde{\mathrm{H}} P^{2}$ from the pseudo-Riemannian metric $g$ on $S^{6,5}$. One can easily check that the endomorphism $J_{1}$ is an isometry and $J_{2}, J_{3}$ are anti-isometries.

We denote the Levi-Civita connection on $S^{6,5}$ by $\stackrel{\varepsilon}{\nabla}$. Let $\bar{\nabla}$ be the induced connection on $\tilde{\mathrm{H}} P^{2}$. The associated curvature tensors are then $\bar{R}$ and $\bar{K}$, respectively. Note that $\bar{K}$ has constant para-quaternionic sectional curvature [3].

\section{The orbit space $\mathscr{K} / G$ and its metric}

Let $p$ and $q$ be positive coprime distinct integers. We define an isometric action $\phi$ of the group $G:=\left\{e^{j t} \mid t \in \mathrm{R}\right\} \cong(\mathrm{R},+)$ on $\tilde{\mathrm{H}} P^{2}$ by setting

$$
\phi_{t}\left(u_{0}, u_{1}, u_{2}\right):=\left(e^{j q t} u_{0}, e^{j p t} u_{1}, e^{j p t} u_{2}\right),
$$

where $e^{j t}:=\cosh t+j \sinh t, t \in \mathrm{R}$. This action preserves the para-quaternionic structure on $\tilde{\mathrm{H}} P^{2}$. The induced Killing vector field at a point $[u]$ of $\tilde{\mathrm{H}} P^{2}$ is

$$
V_{u}:=\pi_{*}\left(j\left(q u_{0}, p u_{1}, p u_{2}\right)\right) .
$$

Let $\mathscr{K}_{0}$ be the algebraic submanifold of $\tilde{\mathrm{H}} P^{2}$ which is defined by the equation

$$
\mathscr{K}_{0}:=\left\{\left[u_{0}, u_{1}, u_{2}\right] \in \tilde{\mathrm{H}} P^{2} \mid q \bar{u}_{0} j u_{0}+p \bar{u}_{1} j u_{1}+p \bar{u}_{2} j u_{2}=0\right\} .
$$

Then $K_{0}$ has real codimension 3 in $\tilde{\mathrm{H}} P^{2}$ so $\operatorname{dim}_{\mathrm{R}} \mathscr{K}_{0}=5$.

Lemma 3.1. The action $\phi_{t}$ of the group $G$ preserves the submanifold $\mathscr{K}_{0}$. It acts freely on $\mathscr{K}_{0}$.

Proof. It is immediate from the definition that the manifold $\mathscr{K}_{0}$ is invariant under the action of $G$. Suppose that the action of $G$ is not free, i.e. that for some $h \in \mathrm{H}_{1}, t \in \mathrm{R}, t \neq 0$ and some $u \in \mathscr{K}_{0} \subset S^{6,5}$ we have

$$
\left(e^{j q t} u_{0}, e^{j p t} u_{1}, e^{j p t} u_{2}\right)=\phi_{t}(u)=u h=\left(u_{0} h, u_{1} h, u_{2} h\right) .
$$

We may then conclude that

$$
\begin{aligned}
0 & =q \bar{u}_{0} j u_{0} h+p \bar{u}_{1} j u_{1} h+p \bar{u}_{2} j u_{2} h \\
& =q \bar{u}_{0} j e^{j q t} u_{0}+p \bar{u}_{1} j e^{j p t} u_{1}+p \bar{u}_{1} j e^{j p t} u_{2} \\
& =\left|u_{0}\right|^{2}(q \sinh q t-p \sinh p t)+p \sinh p t+q(\cosh q t-\cosh p t) \bar{u}_{0} j u_{0} .
\end{aligned}
$$


Hence, we have $q(\cosh q t-\cosh p t) \bar{u}_{0} j u_{0} \in \mathrm{R}$. If $\cosh q t=\cosh p t$ then, since $t \neq 0$, we have $p= \pm q$, which is a contradiction.

Otherwise, we have $\bar{u}_{0} j u_{0}=a \in \mathrm{R}$, If $\left|u_{0}\right|^{2} \neq 0$ then $j=a\left|u_{0}\right|^{-2} \in \mathrm{R}$, which again is a contradiction. If $\left|u_{0}\right|^{2}=0$ then $a \neq 0$, since $p \sinh p t \neq$ 0 , so we may conclude that the action of $G$ is free by computing: $0=$ $\left|u_{0}\right|^{2}|j|^{2}\left|u_{0}\right|^{2}=|a|^{2} \neq 0$.

Since the action $\phi_{t}$ of the group $G$ preserves the algebraic submanifold $\mathscr{K}_{0}$, the vector $V_{u}$ is tangent to $\mathscr{K}_{0}$ at the point $[u] \in \mathscr{K}_{0}$. One can check that the vectors

$$
J_{1} V_{u}, \quad J_{2} V_{u}, \quad J_{3} V_{u} \in T_{[u]} \tilde{\mathrm{H}} P^{2}
$$

are normal to the submanifold $\mathscr{K}_{0}$ at the point $[u]$. At points $[u] \in \mathscr{K}_{0}$ such that $\left|V_{u}\right|^{2} \neq 0$, the vectors $V_{u}, J_{1} V_{u}, J_{2} V_{u}, J_{3} V_{u}$ are linearly independent. Hence, in order to proceed with calculations, we shall restrict to the subset $\mathscr{K}$ of $\mathscr{K}_{0}$ consisting of such points, i.e. to the set

$\mathscr{K}:=\left\{[u]=\left.\left[u_{0}, u_{1}, u_{2}\right] \in \mathscr{K}_{0}\left|n^{2}:=\right| V_{u}\right|^{2}=q^{2}\left|u_{0}\right|^{2}+p^{2}\left|u_{1}\right|^{2}+p^{2}\left|u_{2}\right|^{2} \neq 0\right\}$.

We have the metric $\langle\cdot, \cdot\rangle$ induced from $\tilde{\mathrm{H}} P^{2}$ on $\mathscr{K}$. We shall denote the associated connection, curvature, and sectional curvature by $\stackrel{\circ}{\nabla}, \stackrel{\circ}{R}, \stackrel{\circ}{K}$, respectively. The following lemma is immediate consequence of Lemma 3.1.

Lemma 3.2. $\mathscr{K}$ is a differentiable manifold of real dimension 5. The isometric action $\phi_{t}$ of the group $G$ preserves $\mathscr{K}$ and it is free on $\mathscr{K}$. The quotient manifold $\mathscr{K} / G$ is a pseudo-Riemannian manifold of real signature $(2,2)$.

Let $g_{p, q}$ be the metric induced on $\mathscr{K} / G$ by the Riemannian submersion $\xi$ from $\mathscr{K}$ to $\mathscr{K} / G$. It may be represented by the following diagram.

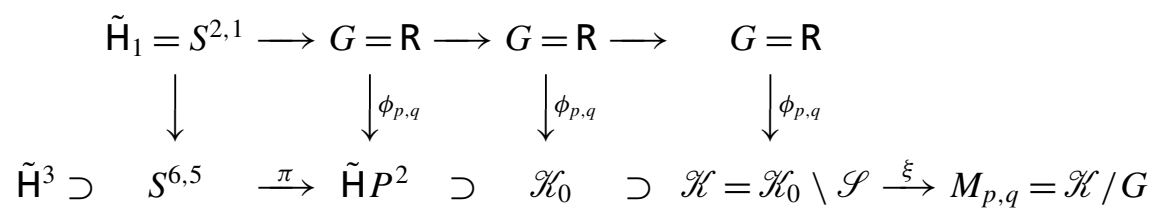

It is not difficult to check that it can be expressed in terms of global coordinates $u \in S^{6,5}$ in the following way

$(\xi \circ \pi)^{*}\left(g_{p, q}\right)=d \bar{u} \otimes_{\mathrm{R}} d u+(d \bar{u} \cdot u) \otimes_{\mathrm{R}}(\bar{u} \cdot d u)+\frac{\left(d \bar{u} \cdot V_{u}\right) \otimes_{\mathrm{R}}\left(\overline{V_{u}} \cdot d u\right)}{\left|V_{u}\right|^{2}}$.

We note that the first two terms determine the metric of the para-quaternionic projective plane. Let $i=1,2,3$. The endomorphisms $J_{i}$ of $T_{[u]} \tilde{\mathrm{H}} P^{n}$ induce 
corresponding endomorphisms $\tilde{J}_{i}$ of $T_{\xi([u])}(\mathscr{K} / G)$. They satisfy the relations given in equation (1). We have:

$$
\tilde{J}_{i}(\tilde{X}, \tilde{Y})=J_{i}(X, Y), \quad \tilde{X}, \tilde{Y} \in T_{\xi([u])}(\mathscr{K} / G),
$$

where $X, Y$ are horizontal lifts of vectors $\tilde{X}, \tilde{Y}$. Moreover, $\tilde{J}_{1}$ is the isometry and $\tilde{J}_{2}, \tilde{J}_{3}$ are the anti-isometries with respect to the metric $g_{p, q}$ which were described previously. Denote the induced connection, curvature and the sectional curvature of $\mathscr{K} / G$ by $\nabla, R$ and $K$, respectively.

\section{The curvature of $\mathscr{K} / G$}

We shall study the curvature tensor of $\mathscr{K} / G$ in this section. Note that the sectional curvature of the quaternionic and the para-quaternionic projective plane can be expressed as

$$
\bar{K}(X, Y)=1+3\left|\operatorname{Pr}_{\left\langle Y, J_{1} Y, J_{2} Y, J_{3} Y\right\rangle} X\right|^{2} / \epsilon(X),
$$

for arbitrary orthonormal tangent vectors $X$ and $Y$, where $\epsilon(X)$ denotes the sign of square length of the vector $X$ (see [3]).

We now compute the connection $\stackrel{\circ}{\nabla}$ and the curvature $\stackrel{\circ}{R}$ of the submanifold $\mathscr{K}_{0}$ of $\tilde{\mathrm{H}} P^{2}$. Let $i$ be the inclusion of $\mathscr{K}$ in $\tilde{\mathrm{H}} P^{2}$. For $[u] \in \mathscr{K}$ we will denote the vectors $X \in T_{[u]} \mathscr{K}$ and $i_{*} X \in T_{[u]} \tilde{\mathrm{H}} P^{2}$ by the same letter $X$. We then have that

$$
\bar{\nabla}_{X} Y=\stackrel{\circ}{\nabla}_{X} Y+B(X, Y), \quad X, Y \in T_{[u]} \mathscr{K},
$$

where $B(X, Y)$ is the second fundamental form at a point $[u] \in \mathscr{K}$. Because we have that $B(X, Y) \in\left(T_{[u]} \mathscr{K}\right)^{\perp}=\mathrm{R}\left\langle J_{1} V_{u}, J_{2} V_{u}, J_{3} V_{u}\right\rangle$,

(4) $\bar{\nabla}_{X} Y-\stackrel{\circ}{\nabla}_{X} Y=B(X, Y)=\sum_{i=1}^{3} \alpha_{i} J_{i} V_{u}$

$$
\text { where } \quad \alpha_{i}=\frac{\epsilon_{i}}{n^{2}}\left\langle\bar{\nabla}_{X} Y, J_{i} V_{u}\right\rangle, \quad i=1,2,3 .
$$

For $i=1,2,3$ we have

$$
\left\langle\bar{\nabla}_{X} Y, J_{i} V_{u}\right\rangle=-\left\langle Y, \bar{\nabla}_{X} J_{i} V_{u}\right\rangle=-\left\langle Y, J_{i} \Lambda X\right\rangle,
$$

where the skew-symmetric operator $\Lambda: T_{[u]} \tilde{\mathrm{H}} P^{2} \rightarrow T_{[u]} \tilde{\mathrm{H}} P^{2}$ is defined by

$$
\Lambda X:=\pi_{*}\left(j\left(q x_{0}, p x_{1}, p x_{2}\right)\right)
$$


here $\left(x_{0}, x_{1}, x_{2}\right)$ is the horizontal lift of $X \in T_{[u]} \tilde{\mathrm{H}} P^{2}$ to $T_{u} S^{6,5}$. Using equations (3), (4) and (5) we finally obtain the relationship

$$
B(X, Y)=-\frac{1}{n^{2}} \sum_{i=1}^{3} \epsilon_{i}\left\langle Y, J_{i} \Lambda X\right\rangle J_{i} V_{u}
$$

Using the relation

$$
\begin{aligned}
\stackrel{\circ}{K}(X, Y) & =\bar{K}(X, Y)-\frac{1}{Q}\left(|B(X, Y)|^{2}-\langle B(X, X), B(Y, Y)\rangle\right), \\
Q & :=Q(X, Y)=|X|^{2}|Y|^{2}-\langle X, Y\rangle^{2},
\end{aligned}
$$

between second fundamental form and sectional curvature, we see that when the sectional curvature $\bar{K}$ is constant that:

$$
\begin{aligned}
& \stackrel{\circ}{K}(X, Y) \\
& \quad=\bar{K}(X, Y)+\frac{1}{n^{2} Q} \sum_{i=1}^{3} \epsilon_{i}\left(-\left\langle Y, J_{i} \Lambda X\right\rangle^{2}+\left\langle X, J_{i} \Lambda X\right\rangle\left\langle Y, J_{i} \Lambda Y\right\rangle\right) .
\end{aligned}
$$

We now use the O'Neill formula to compute the sectional curvature $K$ on $\mathscr{K} / G$ :

(6) $K(\tilde{X}, \tilde{Y})=\stackrel{\circ}{K}(X, Y)+\frac{3}{4} \frac{\langle v[X, Y], v[X, Y]\rangle}{Q(X, Y)}, \quad \tilde{X}, \tilde{Y} \in T_{\xi([u])}(\mathscr{K} / G)$

where $v[X, Y]$ denotes the vertical component of the commutator $[X, Y]$ and $X, Y$ are the unique horizontal lifts of the vectors $\tilde{X}, \tilde{Y}$ to $T_{[u]} \mathscr{K}$. Since the vertical space of the submersion is generated by the vector $V_{u}$, we have

$$
\begin{aligned}
v[X, Y] & =\frac{1}{n^{2}}\left\langle[X, Y], V_{u}\right\rangle V_{u}=\frac{1}{n^{2}}\left(\left\langle\stackrel{\circ}{\nabla}_{X} Y, V_{u}\right\rangle-\left\langle\stackrel{\circ}{\nabla}_{Y} X, V_{u}\right\rangle\right) V_{u} \\
& =\frac{1}{n^{2}}(-\langle Y, \Lambda X\rangle+\langle X, \Lambda Y\rangle) V_{u}=\frac{2}{n^{2}}\langle X, \Lambda Y\rangle V_{u} .
\end{aligned}
$$

We use (6) to see that the sectional curvature of $\mathscr{K} / G$ is given by

$$
\begin{aligned}
& K(\tilde{X}, \tilde{Y})=\bar{K}(X, Y) \\
&+\frac{1}{n^{2} Q}\left[\sum_{i=1}^{3} \epsilon_{i}\left(\left\langle X, J_{i} \Lambda X\right\rangle\left\langle Y, J_{i} \Lambda Y\right\rangle-\left\langle Y, J_{i} \Lambda X\right\rangle^{2}\right)+3\langle X, \Lambda Y\rangle^{2}\right]
\end{aligned}
$$


and $X, Y$ are related to $\tilde{X}, \tilde{Y}$ as above. Then, the Jacobi operator is

$$
\begin{aligned}
& K_{\tilde{X}}(\tilde{Y}):=R(\tilde{X}, \tilde{Y}) \tilde{X}=h(\bar{R}(X, Y) X) \\
& +\frac{1}{n}\left[\sum_{i=1}^{3} \epsilon_{i}\left(-\left\langle X, J_{i} \Lambda X\right\rangle h\left(J_{i} \Lambda Y\right)+\left\langle X, J_{i} \Lambda Y\right\rangle h\left(J_{i} \Lambda X\right)\right)-3\langle Y, \Lambda X\rangle h(\Lambda X)\right],
\end{aligned}
$$

where $h(X)$ denotes the horizontal part of the vector $X \in T_{[u]} \tilde{\mathrm{H}} P^{2}$, i.e. the orthogonal projection of the vector $X$ onto the space $\mathrm{R}\left\langle V_{u}, J_{1} V_{u}, J_{2} V_{u}, J_{3} V_{u}\right\rangle^{\perp}$.

\section{The local geometry of $\mathscr{K} / G$}

In this section we shall use the curvature of $\mathscr{K} / G$ to describe some of the interesting geometric properties of this space.

First, we recall some definitions. Let $R$ be the curvature tensor of a pseudoRiemannian manifold $(M, g)$. The Jacobi operator $K_{X}$ is defined by $K_{X}$ : $Y \rightarrow R(X, Y, X)$. Let $S^{+}(M, g)$ be the bundle of all unit spacelike vectors. The manifold $M$ is said to be pointwise spacelike Osserman if the eigenvalues of the Jacobi operator are constant on $S_{m}^{+}(M, g)$ for every point $m \in M$; the eigenvalues being in principle allowed to vary from point to point. The manifold $M$ is said to be (globally) spacelike Osserman if the eigenvalues constant on $S(M, g)$ and does not vary from point to point. The notions pointwise spacelike Jordan Osserman and spacelike Jordan Osserman are defined similarly using the Jordan normal form rather than the eigenvalue structures.

Lemma 5.1. The manifold $\mathscr{K} / G$ is pointwise spacelike Jordan Osserman of neutral signature.

Proof. Let $X \in T_{[u]} \mathscr{K}$ denote the horizontal lift of a vector $\tilde{X} \in T_{\xi([u])}(\mathscr{K} / G)$. For any unit vector $\tilde{X} \in T_{\xi([u])}(\mathscr{K} / G)$ (i.e. with square norm equals 1$)$ the basis $\left(\tilde{X}, \tilde{J}_{1} \tilde{X}, \tilde{J}_{2} \tilde{X}, \tilde{J}_{3} \tilde{X}\right)$ is pseudo-orthonormal. The vector $\tilde{X}$ is the eigenvector corresponding to the eigenvalue 0 of the self-adjoint operator $K_{\tilde{X}}$. Thus, we are interested only in the restriction of the operator $K_{\tilde{X}}$ to the orthogonal complement $\mathrm{R}\left\langle\tilde{J}_{1} \tilde{X}, \tilde{J} \tilde{X}_{2} \tilde{X}, \tilde{J}_{3} \tilde{X}\right\rangle$ of $\tilde{X}$.

The restriction of $K_{\tilde{X}}$ in the basis $\left(\tilde{J}_{1} \tilde{X}, \tilde{J}_{2} \tilde{X}, \tilde{J}_{3} \tilde{X}\right)$ is represented by the matrix

$$
K=\frac{2}{n^{2}}\left(\begin{array}{ccc}
2 a^{2}+b^{2}+c^{2}-\frac{\bar{c} n^{2}}{2} & -3 a b & -3 a c \\
3 a b & -a^{2}-2 b^{2}+c^{2}-\frac{\bar{c} n^{2}}{2} & -3 b c \\
3 a c & -3 b c & -a^{2}+b^{2}-2 c^{2}-\frac{\bar{c} n^{2}}{2}
\end{array}\right),
$$


where $a, b, c$ are the coordinates of the horizontal component $h(\Lambda X)$ of the vector $\Lambda X$ in the basis $\left(J_{1} X, J_{2} X, J_{3} X\right)$, i.e.

$$
a:=\left\langle X, J_{1} \Lambda X\right\rangle, \quad b:=\left\langle X, J_{2} \Lambda X\right\rangle, \quad c:=\left\langle X, J_{3} \Lambda X\right\rangle,
$$

and $\bar{c}$ is the constant para-quaternionic sectional curvature of the corresponding projective plane. By a direct computation we find that the eigenvalues of the matrix $K$ are

$$
\lambda_{1}=-\frac{2}{n^{2}}\left(-a^{2}+b^{2}+c^{2}\right)+\bar{c}=\lambda_{2}, \quad \lambda_{3}=\frac{4}{n^{2}}\left(-a^{2}+b^{2}+c^{2}\right)+\bar{c} .
$$

To show that the space $\mathscr{K} / G$ is the pointwise Osserman we have to show that the eigenvalues $\lambda_{i}$ are independent of the chosen unit direction $\tilde{X}$, i.e. of its horizontal unit lift $X \in T_{[u]} \mathscr{K}$. Notice that $-a^{2}+b^{2}+c^{2}=|h(\Lambda X)|^{2}$. Let $e \in T_{[u]} \mathscr{K},|e|^{2}=1$ be a fixed unit vector and $\left(e, J_{1} e, J_{2} e, J_{3} e\right)$ the corresponding pseudo-orthonormal basis. Any horizontal unit vector $X$ can be written in the form

$$
X=X_{0} e+X_{1} J_{1} e+X_{2} J_{2} e+X_{3} J_{3} e, \quad X_{0}^{2}+X_{1}^{2}-X_{2}^{2}-X_{3}^{2}=1 .
$$

Now one can check directly that

$$
-a^{2}+b^{2}+c^{2}=|h(\Lambda X)|^{2}=|h(\Lambda e)|^{2}=\mathrm{const}
$$

and hence the eigenvalues of the Jacobi operator $K_{\tilde{X}}$ are constant at a given point. Moreover, one can check that the Jacobi operator is diagonalizable; this completes the proof of the lemma.

THEOREM 5.2. The manifold $\mathscr{K} / G$ is Einstein, self-dual and not locally homogenous of the signature $(--++)$.

Proof. In [2], the self-dual, Einstein manifolds of neutral signature are characterized as the pointwise-Osserman manifolds. Thus Lemma 5.1 implies that $\mathscr{K} / G$ is Einstein and self-dual. A pointwise-Osserman, locally homogenous manifold is Osserman, i.e. the eigenvalues its Jacobi operator is also independent of the point. But for our examples, we can check that the eigenvalues of the Jacobi operator, $\lambda_{1}, \lambda_{2}$ and $\lambda_{3}$ are are not constant on $\mathscr{K} / G$. After some computations, where a few unexpected cancelations happened, we obtain

$$
\lambda_{1}=\lambda_{2}=-\frac{2 p^{4} q^{2}}{n^{6}}-4, \quad \lambda_{3}=\frac{4 p^{4} q^{2}}{n^{6}}-4
$$


Clearly, $n^{2}$ is not a constant along $\mathscr{K} / G(\mathscr{K} / G$ is not globally Osserman). Thus, the manifold $\mathscr{K} / G$ is not locally homogeneous, and hence not locally symmetric.

REMARK. It is interesting that there exist a three-dimensional submanifold of $\mathscr{K} / G$ along which the eigenvalues of the Jacobi operator are constant.

REMARK. In the case $p=1=q$, there are no singular points, so $\mathscr{K}=\mathscr{K}_{0}$. Since the action of the group $G$ on $\mathscr{K}$ is free, the resulting space $\mathscr{K} / G$ is a complete manifold of neutral signature. One can check that vector $\Lambda X, X \in$ $T_{[u]} \mathscr{K}$ is horizontal and $\bar{c}=4$, so $\lambda_{1}=2=\lambda_{2}, \lambda_{3}=8$, i.e. the eigenvalues of the Jacobi operator are constant on $\mathscr{K} / G$. This implies that $M_{1,1}=\mathscr{K} / G$ is Einstein, self-dual, globally Osserman neutral manifold. Moreover, this manifold is isometric to para-complex projective plane, where para-complex stucture is induced by the left multiplication by the generator $j$ (see [4]).

\section{Study of the example of Galicki and Lawson}

We return to the starting point for our work, the compact Riemannian orbifolds

$$
\mathcal{O}_{q, p}=\mathscr{L}_{H} / S^{1},
$$

constructed and studied by Galicki and Lawson [7]. In this section we will discuss some additional properties which concern local geometry and curvature of the spaces $\mathscr{O}_{p, q}(1)$. We will see in particular that the orbifolds $\mathscr{O}_{p, q}(1)$ are not locally homogeneous for any $p, q$. We shall also see that the orbifolds have positive sectional curvature for some values of $p, q$. Finally, we shall give an estimate of the pinching constant, i.e. the ratio of the minimal and maximal sectional curvature.

In [7] was shown that $\mathscr{O}_{q, p}(n-1)$ is not locally symmetric for $0<q / p<1$. Here, for $n=2$ we will give another proof of this result based on the study of the curvature operators along the lines performed in Section §5. This approach provides an opportunity to see that $\mathscr{O}_{p, q}(1)$ is not even locally homogeneous.

TheOREM 6.1 ([7]). The orbifold $\mathscr{O}_{q, p}=\mathscr{L}_{H} / S^{1}$ is an Einstein, self-dual manifold which is not locally homogeneous for any $p, q,(p, q) \neq 1$.

Proof. The proof is based on the characterization of the Einstein, selfdual manifolds as manifolds with constant eigenvalues of the Jacobi operators (pointwise Osserman manifolds), obtained by Vanhecke and Sekigawa [10]. Repeating the long and nice computations from the neutral signature case (under some minor modifications), we express the eigenvalues of the Jacobi 
operator, $K_{\tilde{X}}: Y \rightarrow R(\tilde{X}, Y) \tilde{X}$, in very simply way

$$
\lambda_{1}=\lambda_{2}=\frac{2 p^{4} q^{2}}{\left|V_{u}\right|^{6}}-4, \quad \lambda_{3}=-\frac{4 p^{4} q^{2}}{\left|V_{u}\right|^{6}}-4
$$

at the orbit determined by $[u] \in \mathscr{L}_{H}$ and the arbitrary unit tangent vector $\tilde{X}$. In this computations operator $\Lambda: T_{[u]} \tilde{\mathrm{H}} P^{2} \rightarrow T_{[u]} \tilde{\mathrm{H}} P^{2}$ plays an important role. It is important to notice that we express the eigenvalues in the terms of the quaternionic notation. By the argument as in the proof of Theorem 5.2 proof is completed.

We may now study the cases when the sectional curvature of the orbifold $\mathcal{O}_{p, q}(1)$ is positive.

LEMMA 6.2. Let $\tilde{X}$ and $\tilde{Y}$ the unit orthogonal tangent vectors. For the sectional curvature $K(\tilde{X}, \tilde{Y})$ of the orbifold $\mathcal{O}_{p, q}(1)$, one has the pinching estimates:

$$
\begin{aligned}
& 4-\frac{2 q^{2}}{p^{2}} \leq K(\tilde{X}, \tilde{Y}) \leq 4+\frac{4 q^{2}}{p^{2}} \quad \text { for } \quad p \leq q, \\
& 4-\frac{2 p^{4}}{q^{4}} \leq K(\tilde{X}, \tilde{Y}) \leq 4+\frac{4 p^{4}}{q^{4}} \quad \text { for } \quad p \geq q .
\end{aligned}
$$

Proof. For $p \leq q$ we have $p^{2} \leq|\Lambda u|^{2} \leq q^{2}$ and

$$
-\lambda_{1} \geq 4-\frac{2 q^{2}}{p^{2}}, \quad-\lambda_{3} \leq 4+\frac{4 q^{2}}{p^{2}} .
$$

Since $-\lambda_{1} \leq K(x, y) \leq-\lambda_{3}$, the relation given in equation (9) holds. Similarly, for $p \geq q$ we have $q^{2} \leq|\Lambda u|^{2} \leq p^{2}$ and

$$
-\lambda_{1} \geq 4-\frac{2 p^{4}}{q^{4}}, \quad-\lambda_{3} \leq 4+\frac{4 p^{4}}{q^{4}} .
$$

As in the previous case this completes the proof the Lemma.

We can draw the following consequences of these computations:

THEOREM 6.3. If $p^{2}<\sqrt{2} q^{2}<2 \sqrt{2} p^{2}$, then the orbifold $\mathscr{O}_{p, q}(1)$ has the positive sectional curvature 
Lemma 6.4. For $p^{2}<\sqrt{2} q^{2}<2 \sqrt{2} p^{2}$, let the sectional curvature $K(\tilde{X}, \tilde{Y})$ of the orbifold $\mathcal{O}_{p, q}(1)$ is $k$-pinched, $0<k<1$. Then at an arbitrary point

$$
\begin{aligned}
& \frac{1}{4}-\frac{3}{4} \frac{q^{2}-p^{2}}{p^{2}+q^{2}} \leq k \leq \frac{1}{4}+\frac{3}{4} \frac{q^{4}-p^{4}}{p^{4}+q^{4}} \quad \text { for } \quad p \leq q, \\
& \frac{1}{4}-\frac{3}{4} \frac{p^{4}-q^{4}}{p^{4}+q^{4}} \leq k \leq \frac{1}{4}+\frac{3}{4} \frac{p^{2}-q^{2}}{p^{2}+q^{2}} \quad \text { for } \quad p \geq q .
\end{aligned}
$$

For $p \rightarrow q, k \rightarrow 1 / 4$. Moreover, $k=1 / 4$ on $\mathcal{O}_{p, q}(1)$ if and only if $p=q=1$.

REMARK. The orbifold $\mathscr{O}_{1,1}(1)$ is globally Osserman and has constant holomorphic sectional curvature with respect to the complex structure induced by the left multiplication by $j$ (see [5]).

\section{REFERENCES}

1. Alekseevsky, D. V., Compact quaternionic spaces, Functional Anal. Appl. 2, 11 (1968), 114 116.

2. Alekseevsky, D. V., Blažić, N., Bokan, N., and Rakić, Z., Self-duality and pointwise Osserman condition, Arch. mathematicum 3(35) (1999), 193-201.

3. Blažić, N., Para-quaternionic projective space and pseudo-Riemannian geometry, Publ. Inst. Math. (Beograd) (N.S.) 60(74) (1996), 101-107.

4. Blažić, N., Bokan, N., and Rakić, Z., Characterization of 4-dimensional Osserman pseudoRiemannian manifolds, Bull. Austral. Math. Soc. 3 (2001), 367-395.

5. Chi, Q. S., A curvature characterization of certain locally rank-one symmetric spaces, J. Differential Geom. 28 (1988), 187-202.

6. Galicki, K., A generalization of the momentum mapping construction for quaternionic Kähler manifolds, Comm. Math. Phys. 108 (1987), 117-138.

7. Galicki, K., and Lawson B., Quaternionic reduction and quaternionic orbifolds, Math. Ann. 282 (1988), 1-21.

8. Kamada, H., and Machida, Y., Self-duality of type $(2,2)$ on four-dimensional manifolds, Tohôku Math. J. 49 (1997), 259-275.

9. Swann, A., HyperKähler and quaternionic Kähler geometry, Math. Ann. 289 (1991), 421450.

10. Sekigawa, K., and Vanhecke, L., Volume preserving geodesic symmetries on four dimensional Kähler manifolds, Differential geometry Penścola, Proceedings (A. M. Naveira, A. Fernandez and F. Mascaro, eds.), Lecture Notes in Math. 1209 (1985), 275-290.

FACULTY OF MATHEMATICS

UNIVERSITY OF BELGRADE

STUDENSKI TRG 16

P.P. 550

11000 BELGRADE

YUGOSLAVIA

E-mail: blazicn@matf.bg.ac.yu,vsrdjan@matf.bg.ac.yu 